\title{
An efficient One-Pot Approach to the Synthesis of Symmetric Trithiocarbonates from Carbon Disulfide and Alkyl Halides Using Imidazole
}

\author{
Mohammad Soleiman-Beigi, ${ }^{, a}$ Zahra Taherinia \\ a Department of Chemistry, Faculty of Basic Sciences, Ilam University, P. O. Box 69315-516, Ilam, Iran. \\ Fax: +98(841)2227022; E-mail: SoleimanBeigi@yahoo.com
}

Genera procedures for the synthesis of symmetrical trithiocarbonates from alkyl halides and carbon disulfide:

Alkyl halides $(2.0 \mathrm{mmol})$ (or alkyl dihalides $(1.0 \mathrm{mmol})$ ) were added in one portion to a solution of imidazole $(6.0 \mathrm{mmol})$ and $\mathrm{CS}_{2}(6.0 \mathrm{mmol})$ in $5 \mathrm{~mL} \mathrm{DMSO} / \mathrm{H}_{2} \mathrm{O}(4.8 / 0.2)$. The color of the resulting reaction solution immediately changed from light red to yellow. Then, the solution was allowed to stir for the appropriate time (Table 2) at $45{ }^{\circ} \mathrm{C}$ in air atmosphere. The progress of the reaction was monitored by TLC. After completion of the reaction, $20 \mathrm{ml}$ $\mathrm{CH}_{2} \mathrm{Cl}_{2}$ was added to the mixture, and the mixture was then washed with water $(2 \times 20 \mathrm{ml})$. The organic layer was separated and then dried over anhydrous $\mathrm{Na}_{2} \mathrm{SO}_{4}$. The solvent was evaporated under the reduced pressure. The residue was purified by preparative TLC (silica gel, eluent $n$-hexane: EtOAc; 30:1) to afford pure product (symmetric dialkyl trithiocarbonate). In the case of entries 10 and 11 , table $2(\mathbf{6 j}$ and $\mathbf{6 k})$ the TLC eluent was $n$ hexane: EtOAc 4:1.

Table 2, Entry2 (6b): yellow oil<smiles>S=C(SCc1ccccc1)SCc1ccccc1</smiles>

${ }^{1} \mathrm{H} \mathrm{NMR}\left(400 \mathrm{MHz}, \mathrm{CDCl}_{3}\right): \delta=3.0(\mathrm{~s}, 4 \mathrm{H}), 7.4(\mathrm{~m}, 10 \mathrm{H})$.

${ }^{13} \mathrm{C}$ NMR $\left(100 \mathrm{MHz}, \mathrm{CDCl}_{3}\right): \delta=41.4,127.9,121.6,129.3,133.2,135.0,222.7$.

Table 2, Entry5 (6e): pale yellow oil<smiles>CCC(C)SC(=S)SC(C)CC</smiles> 


\section{Supporting Information}

${ }^{1} \mathrm{H} \mathrm{NMR}\left(400 \mathrm{MHz}, \mathrm{CDCl}_{3}\right): \delta=1.01(\mathrm{t}, J=7.2 \mathrm{~Hz}, 3 \mathrm{H}), 1.4(\mathrm{~d}, J=6.8 \mathrm{~Hz}, 12 \mathrm{H}), 1.65-1.83$ (m, 2H.), 4.21(sext, $J=6.8 \mathrm{~Hz}, 2 \mathrm{H})$.

${ }^{13} \mathrm{C} \mathrm{NMR}\left(100 \mathrm{MHz}, \mathrm{CDCl}_{3}\right): \delta=11.4,19.6,23.2,28.8,47.9,224.1$.

Table 2, Entry6 (6f): pale yellow oil<smiles>CC(C)SC(=S)SC(C)C</smiles>

${ }^{1} \mathrm{H} \mathrm{NMR}\left(400 \mathrm{MHz}, \mathrm{CDCl}_{3}\right): \delta=1.41(\mathrm{~d}, J=6.8 \mathrm{~Hz}, 12 \mathrm{H}), 4.21(\mathrm{sept}, J=7.2 \mathrm{~Hz}, 2 \mathrm{H})$.

${ }^{13} \mathrm{C} \mathrm{NMR}\left(100 \mathrm{MHz}, \mathrm{CDCl}_{3}\right): \delta=22.0,41,6,223.6$.

Table 2, Entry7 (6g): yellow solid, $\mathrm{M} p=64-65^{\circ} \mathrm{C}$<smiles>CC(C)(C)SC(=S)SC(C)(C)C</smiles>

${ }^{13} \mathrm{C}$ NMR $\left(100 \mathrm{MHz}, \mathrm{CDCl}_{3}\right): \delta=14.1,20.72,223.3$.

MS (EI , $70 \mathrm{eV}): \mathrm{m} / \mathrm{z}=57.1,64,71.2,83.0,91.2,111.2,123.2,158.2,168.1,223.9$.

Table 2, Entry4 (6h): yellow oil<smiles>S=C(SCCc1ccccc1)SCCC1C=CC=CC1</smiles>

${ }^{1} \mathrm{H} \mathrm{NMR}\left(400 \mathrm{MHz}, \mathrm{CDCl}_{3}\right): \delta=3.05(\mathrm{t}, J=7.8 \mathrm{~Hz}, 4 \mathrm{H}), 3.67(\mathrm{t}, J=7.8 \mathrm{~Hz}, 4 \mathrm{H}), 7.26-$ 7.37(m, 10H).

${ }^{13} \mathrm{C} \mathrm{NMR}\left(100 \mathrm{MHz}, \mathrm{CDCl}_{3}\right): \delta=34.5,37.9,126.7,128.6,128.6,139.6,223.7$.

MS (EI , $70 \mathrm{eV}): m / z=51,65,77,91,104.1,135,214.1,319.1$.

Table 2, Entry 9 (6i): yellow oil<smiles>S=C(SCCCc1ccccc1)SCCc1ccccc1</smiles> 


\section{Supporting Information}

${ }^{1} \mathrm{H} \mathrm{NMR}\left(400 \mathrm{MHz}, \mathrm{CDCl}_{3}\right): \delta=2.1$ (quin, $\left.J=7.4 \mathrm{~Hz}, 4 \mathrm{H}\right), 2.79(\mathrm{t}, J=7.6 \mathrm{~Hz}, 4 \mathrm{H}), 3.4(\mathrm{t}$, $J=7.4 \mathrm{~Hz}, 4 \mathrm{H}), 7.26-7.38$ (m, 10H).

${ }^{13} \mathrm{C} \mathrm{NMR}\left(100 \mathrm{MHz}, \mathrm{CDCl}_{3}\right): \delta=29.7,34.9,36.1,126.2,129.5,140.9,224.1$.

MS (EI , $70 \mathrm{eV}): m / z=65.1,91.1,117.2,151.1,195.1,313.2,347.3$.

Table 2, Entry $10(6 \mathbf{6 j})$ : Pale yellow oil<smiles>CCOC(=O)CSC(=S)SCC(=O)OCC</smiles>

${ }^{1} \mathrm{H} \mathrm{NMR}\left(400 \mathrm{MHz}, \mathrm{CDCl}_{3}\right): \delta=1.32(\mathrm{t}, J=7.2 \mathrm{~Hz}, 6 \mathrm{H}), 4.20(\mathrm{~s}, 4 \mathrm{H}), 4.26(\mathrm{q}, J=7.0 \mathrm{~Hz}, 4 \mathrm{H})$.

${ }^{13} \mathrm{C}$ NMR $\left(100 \mathrm{MHz}, \mathrm{CDCl}_{3}\right): \delta=14.1,38.9,62.2,167.1,220.3$.

MS (EI , $70 \mathrm{eV}): m / z=59.1,87.1,107,119,135,140.1,168,195,206,237.1,282.1$.

Table 2, Entry12 (61): yellow oil<smiles>C=CCSC(=S)SCC=C</smiles>

${ }^{1} \mathrm{H} \mathrm{NMR}\left(400 \mathrm{MHz}, \mathrm{CDCl}_{3}\right): \delta=4.1(\mathrm{~d}, J=6.8 \mathrm{~Hz}, 4 \mathrm{H}), 5.20(\mathrm{dd}, J=10.4,0.8 \mathrm{~Hz}, 2 \mathrm{H})$, $5.36(\mathrm{dd}, J=17.2,1.2 \mathrm{~Hz}, 2 \mathrm{H}), 5.93(\mathrm{~m}, 2 \mathrm{H})$.

${ }^{13} \mathrm{C}$ NMR $\left(100 \mathrm{MHz}, \mathrm{CDCl}_{3}\right): \delta=39.1,119.6,130,7,222.4$.

Table 3, Entry 3 (8c): yellow oil<smiles>S=C1SCCCCS1</smiles>

${ }^{1} \mathrm{H} \mathrm{NMR}\left(400 \mathrm{MHz}, \mathrm{CDCl}_{3}\right): \delta=2.51(\mathrm{t}, J=2.0 \mathrm{~Hz}, 4 \mathrm{H}), 3.29(\mathrm{t}, J=2.0 \mathrm{~Hz}, 4 \mathrm{H})$.

${ }^{13} \mathrm{C} \mathrm{NMR}\left(100 \mathrm{MHz}, \mathrm{CDCl}_{3}\right): \delta=29.72,38.69,223.32$.

MS (EI , $70 \mathrm{eV}): m / z=54.1,60.1,76,88.1,120.1,164.1$.

Product 9: pale yellow oil 


\section{Supporting Information}<smiles>CCOC(=O)CSC(=S)SCC</smiles>

${ }^{1} \mathrm{H} \mathrm{NMR}\left(400 \mathrm{MHz}, \mathrm{CDCl}_{3}\right): \delta=1.3(\mathrm{t}, J=7.2 \mathrm{~Hz}, 3 \mathrm{H}), 1.41(\mathrm{t}, J=7.4 \mathrm{~Hz}, 3 \mathrm{H}), 3.4(\mathrm{q}, J=7.4$ $\mathrm{Hz}, 2 \mathrm{H}), 4.20$ (s, 2H), 4.26(q, J=7.2 Hz, 2H).

${ }^{13} \mathrm{C}$ NMR $\left(100 \mathrm{MHz}, \mathrm{CDCl}_{3}\right): \delta=12.9,14.1,31.8,38.4,62.1,167.5,222.2$.

MS (EI , $70 \mathrm{eV}): m / z=61,77,106,120,135,163,179,195,224.1$.

Intermediate I: yellow oil<smiles>S=C(SCc1ccccc1)n1ccnc1</smiles>

${ }^{1} \mathrm{H} \mathrm{NMR}\left(400 \mathrm{MHz}, \mathrm{CDCl}_{3}\right): \delta=4.64(\mathrm{~s}, 2 \mathrm{H}), 7.14(\mathrm{~s}, 1 \mathrm{H}), 7.29-7.43(\mathrm{~m}, 5 \mathrm{H}), 7.8(\mathrm{~s}, 1 \mathrm{H}), 8.52$ $(\mathrm{s}, 1 \mathrm{H})$.

${ }^{13} \mathrm{C} \mathrm{NMR}\left(100 \mathrm{MHz}, \mathrm{CDCl}_{3}\right): \delta=41.8,117.7,128.3,128.9,129.4,131.5,133.5,135.7,197.3$. MS (EI , $70 \mathrm{eV}): m / z=50,57.1,65.2,76.1,83.1,91.2,111.2,123.2,158.2,168.1,234.1$. 
Supporting Information

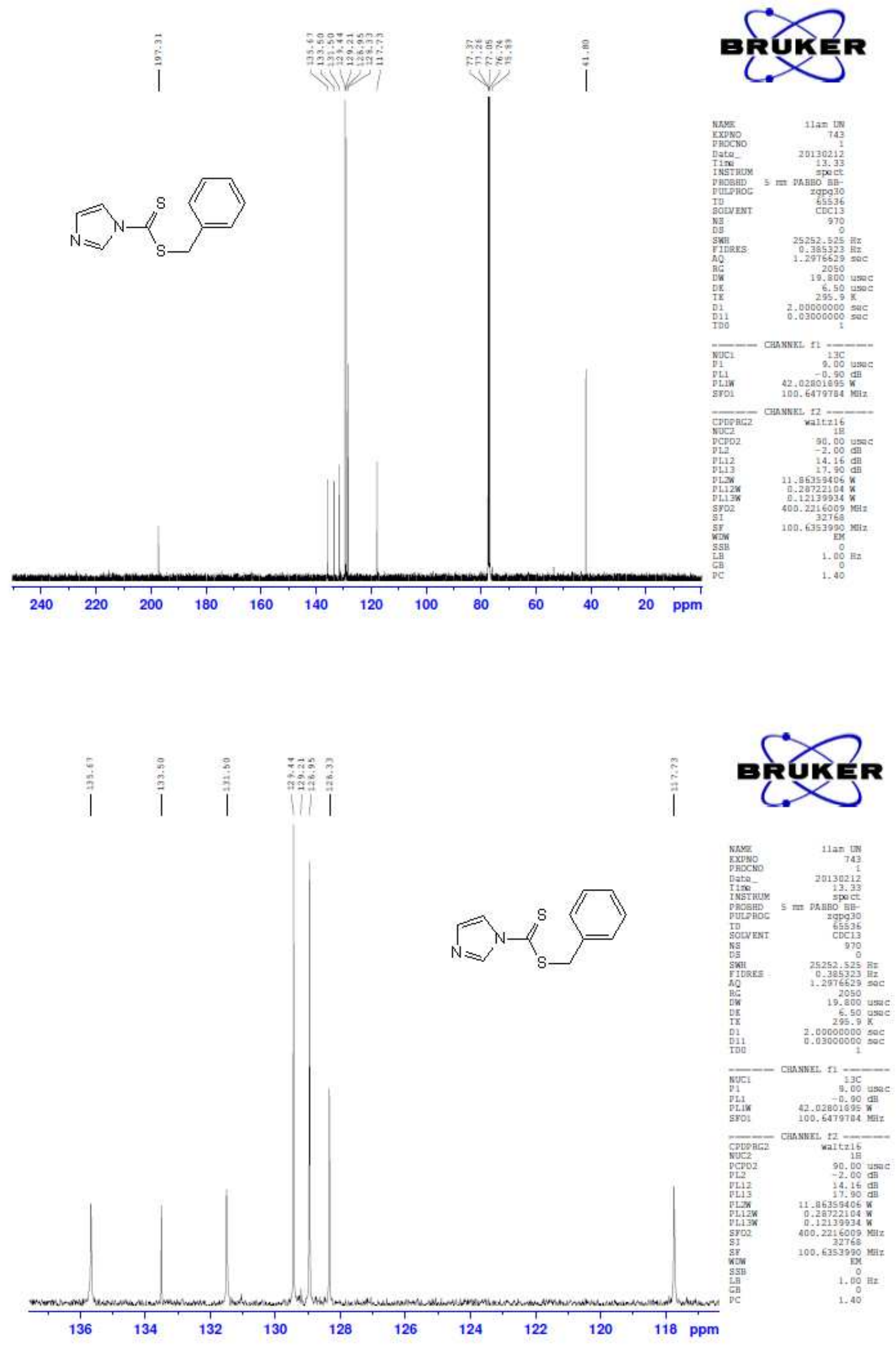




\section{Supporting Information}
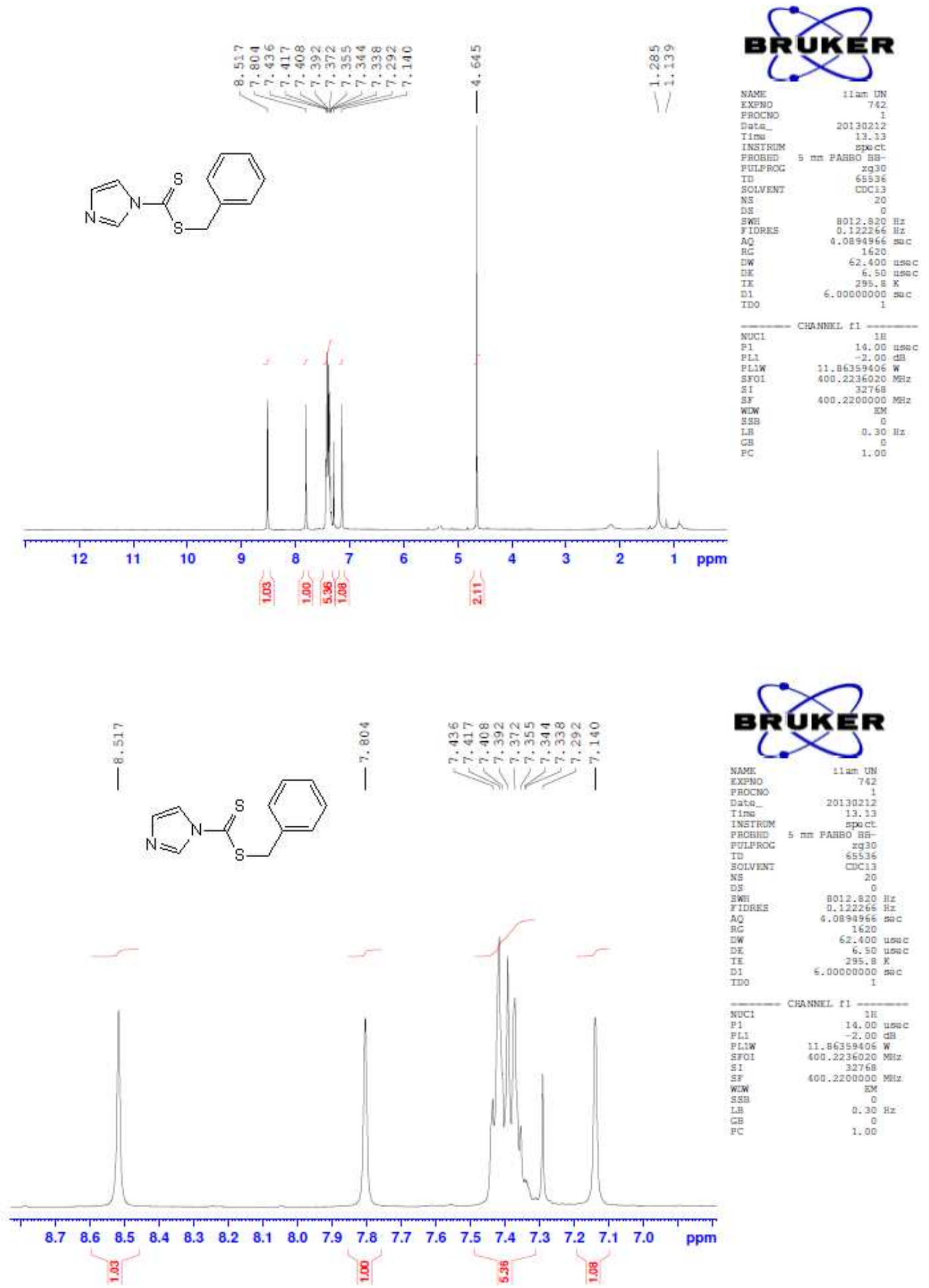
Supporting Information

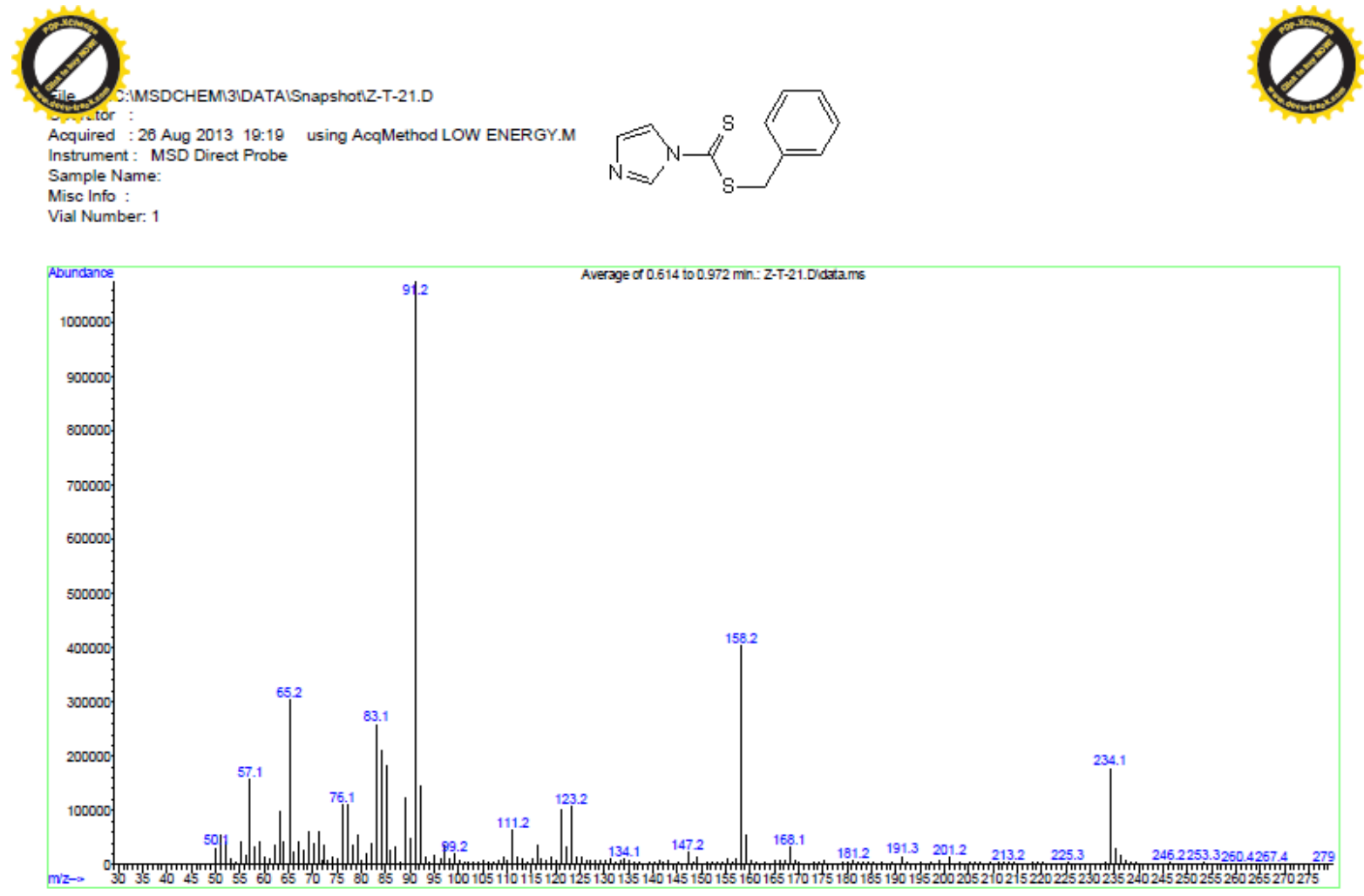


Supporting Information

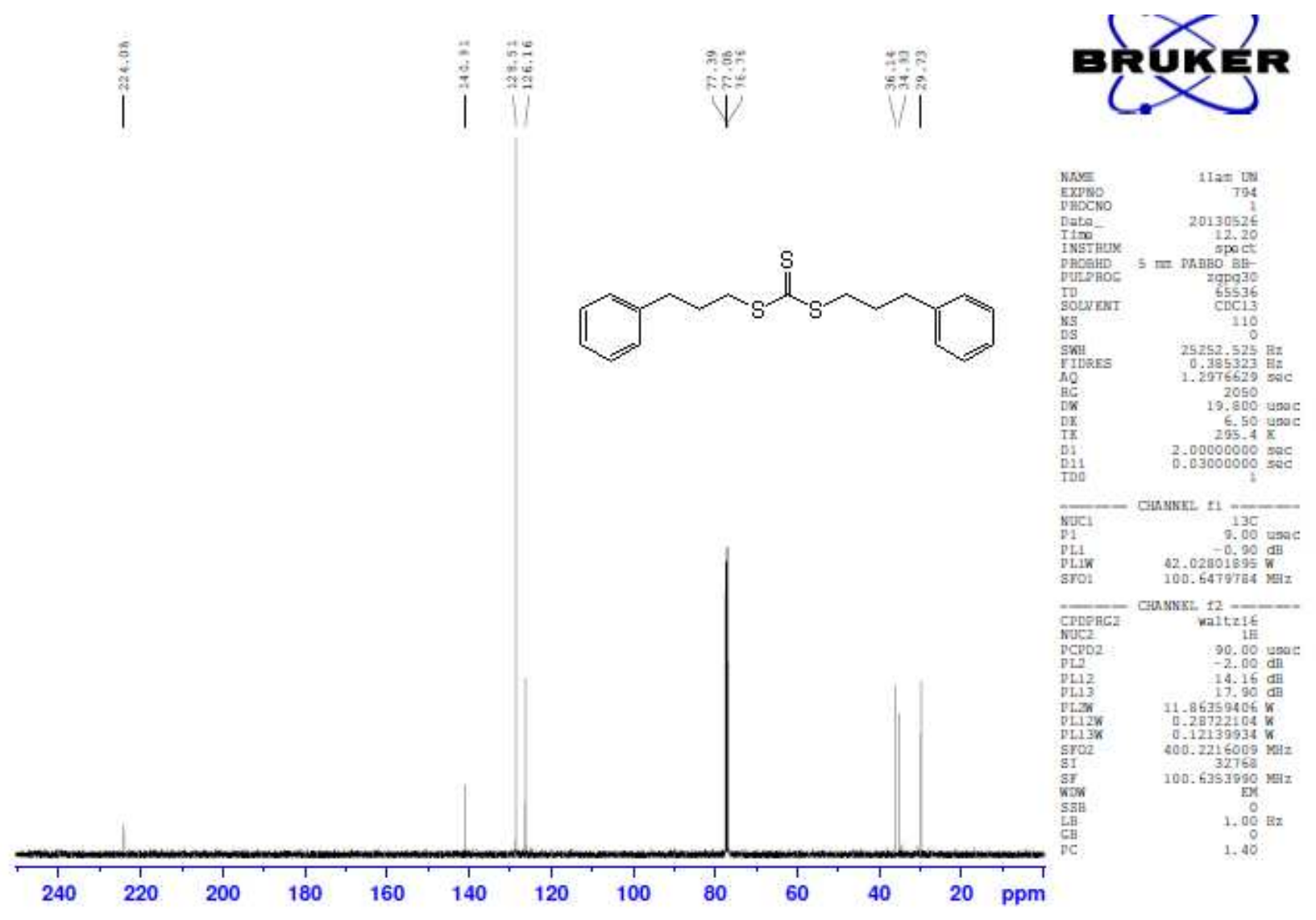


Supporting Information
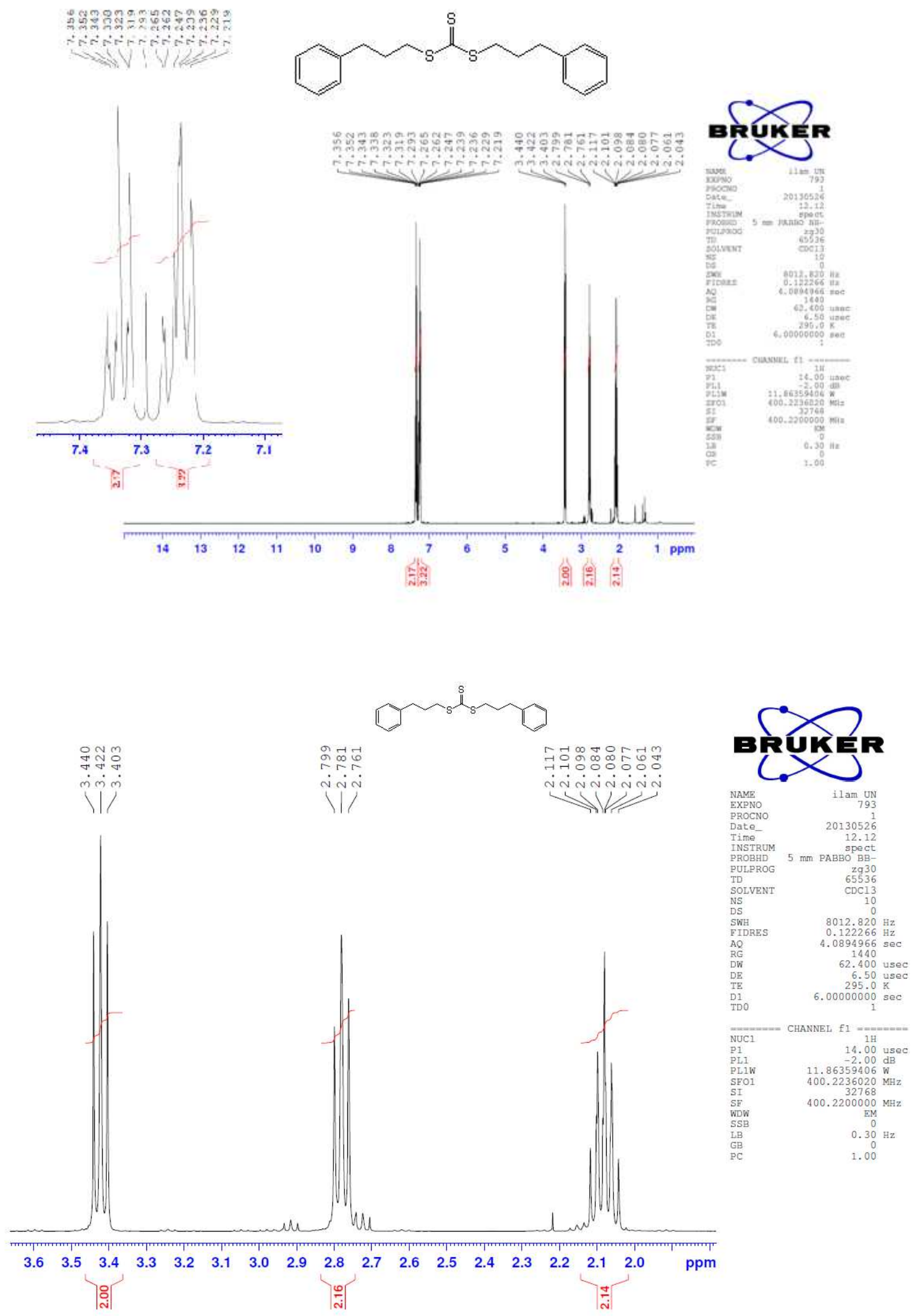
Supporting Information

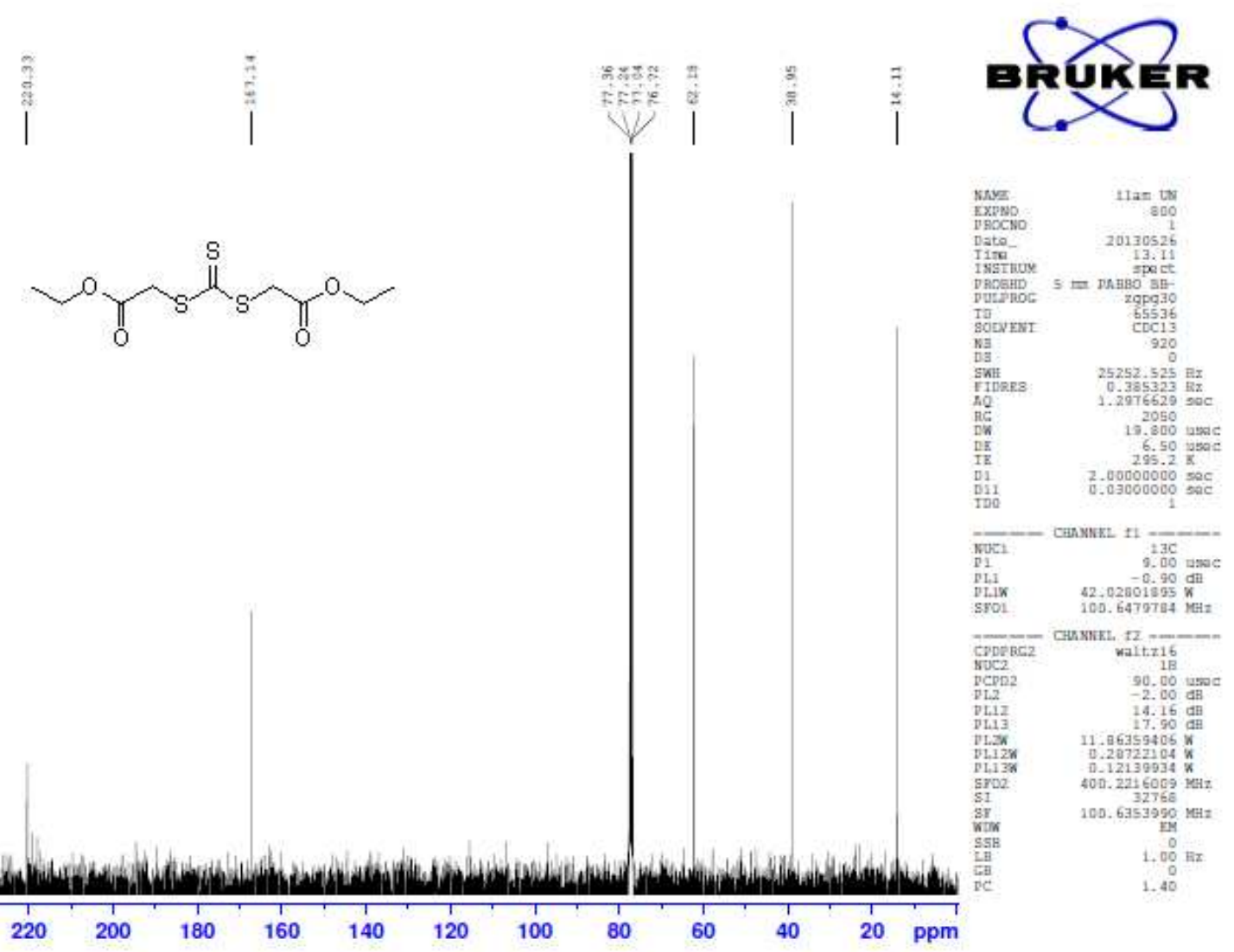




\section{Supporting Information}

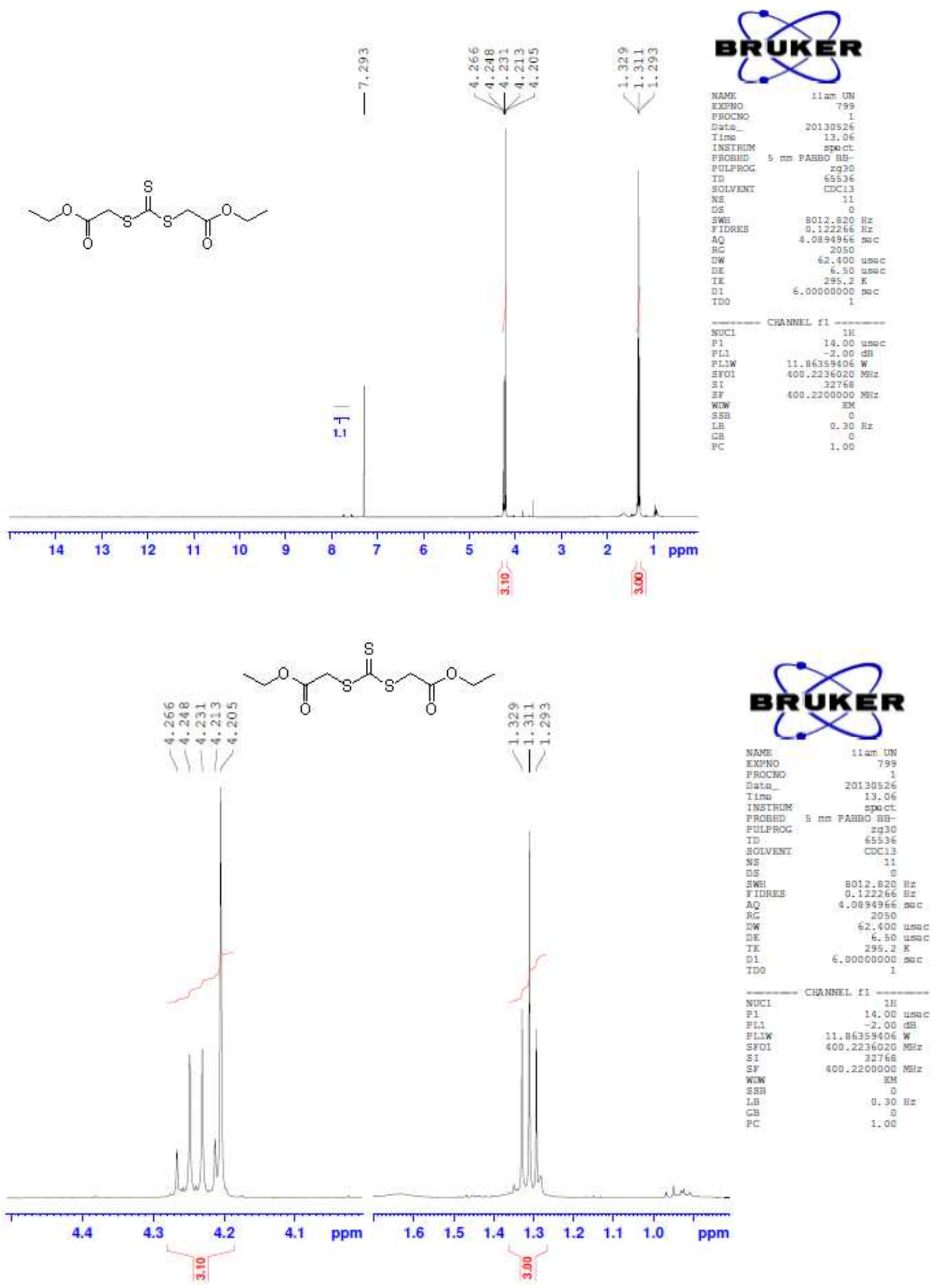


Supporting Information
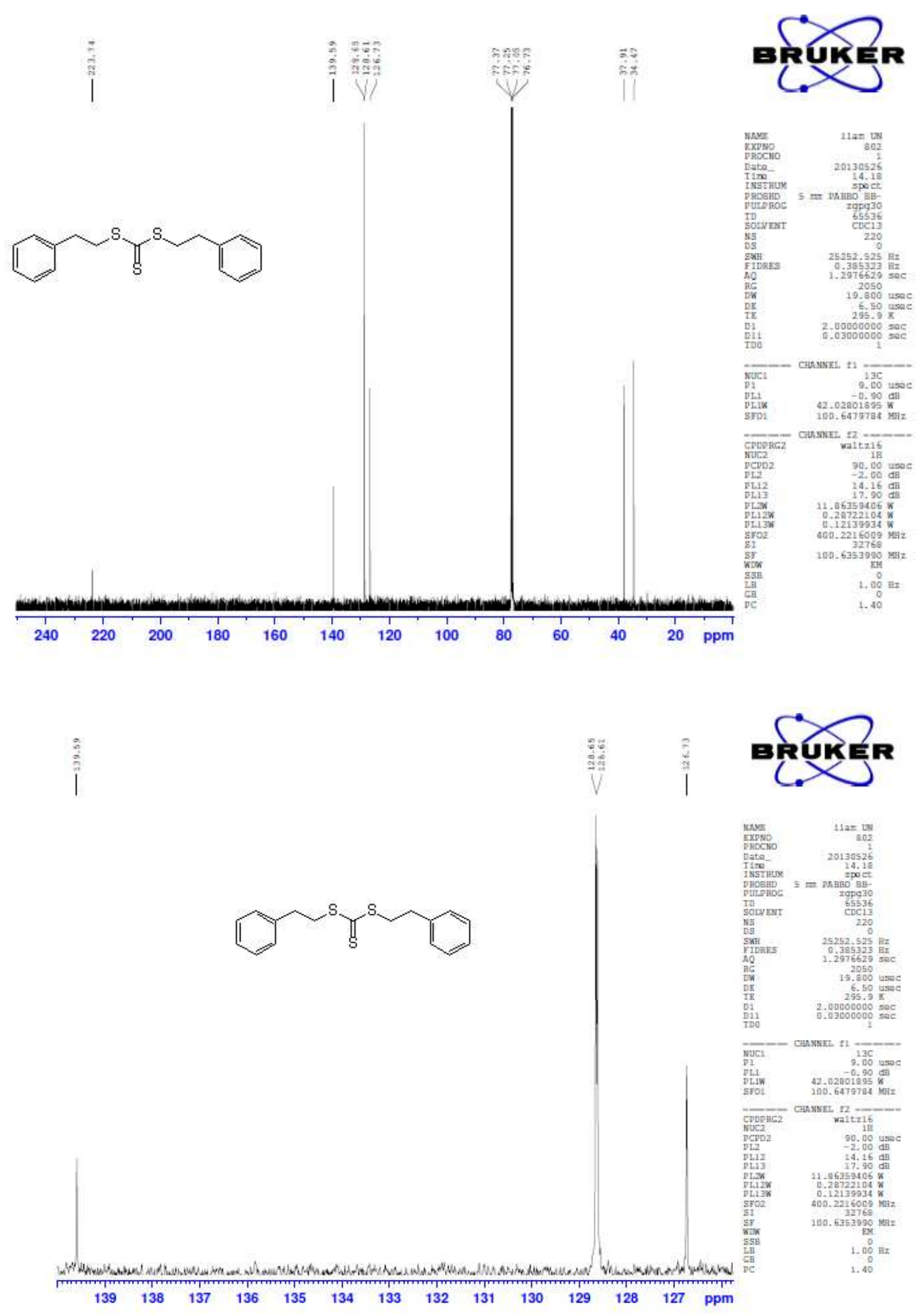
Supporting Information
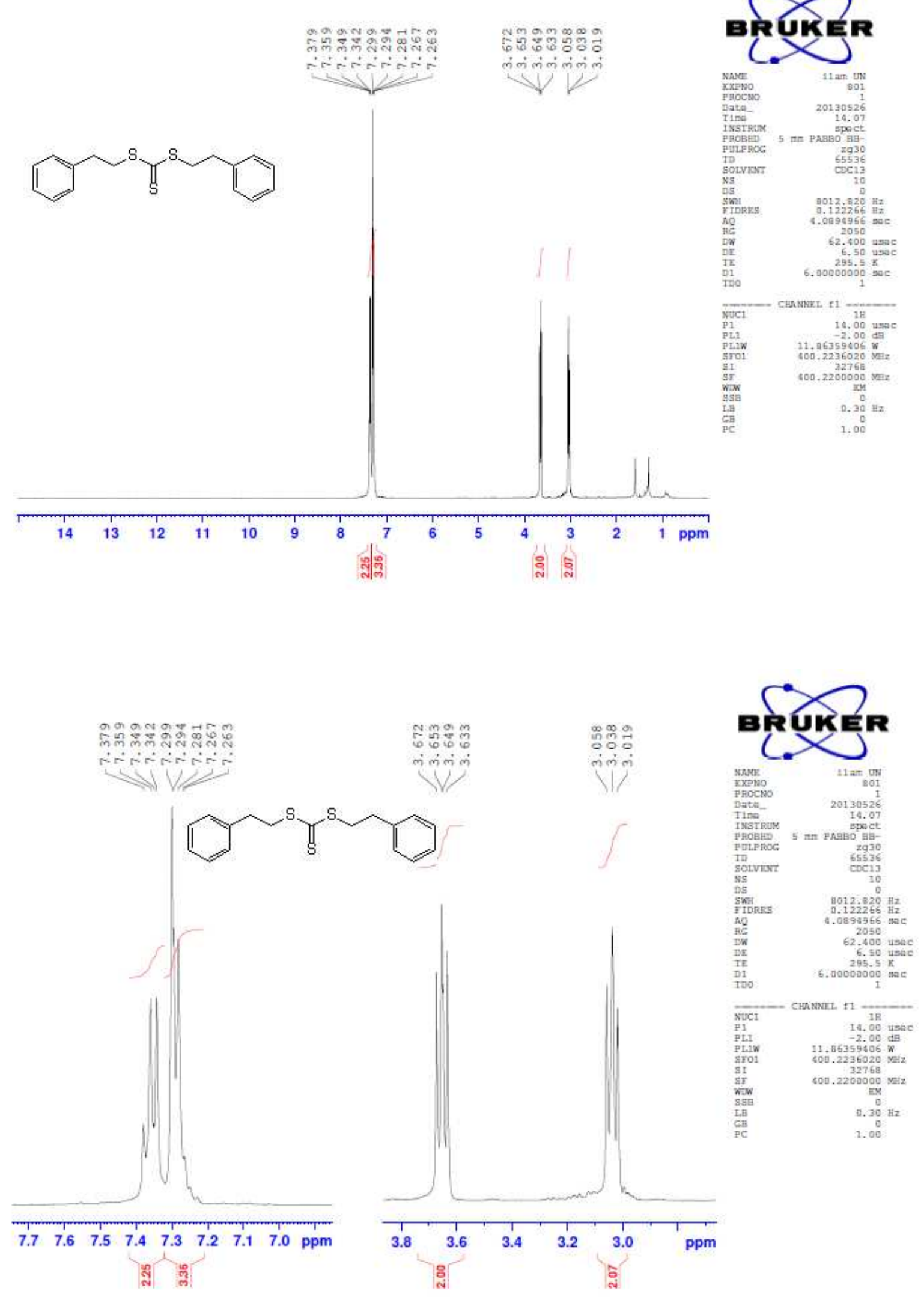
Supporting Information

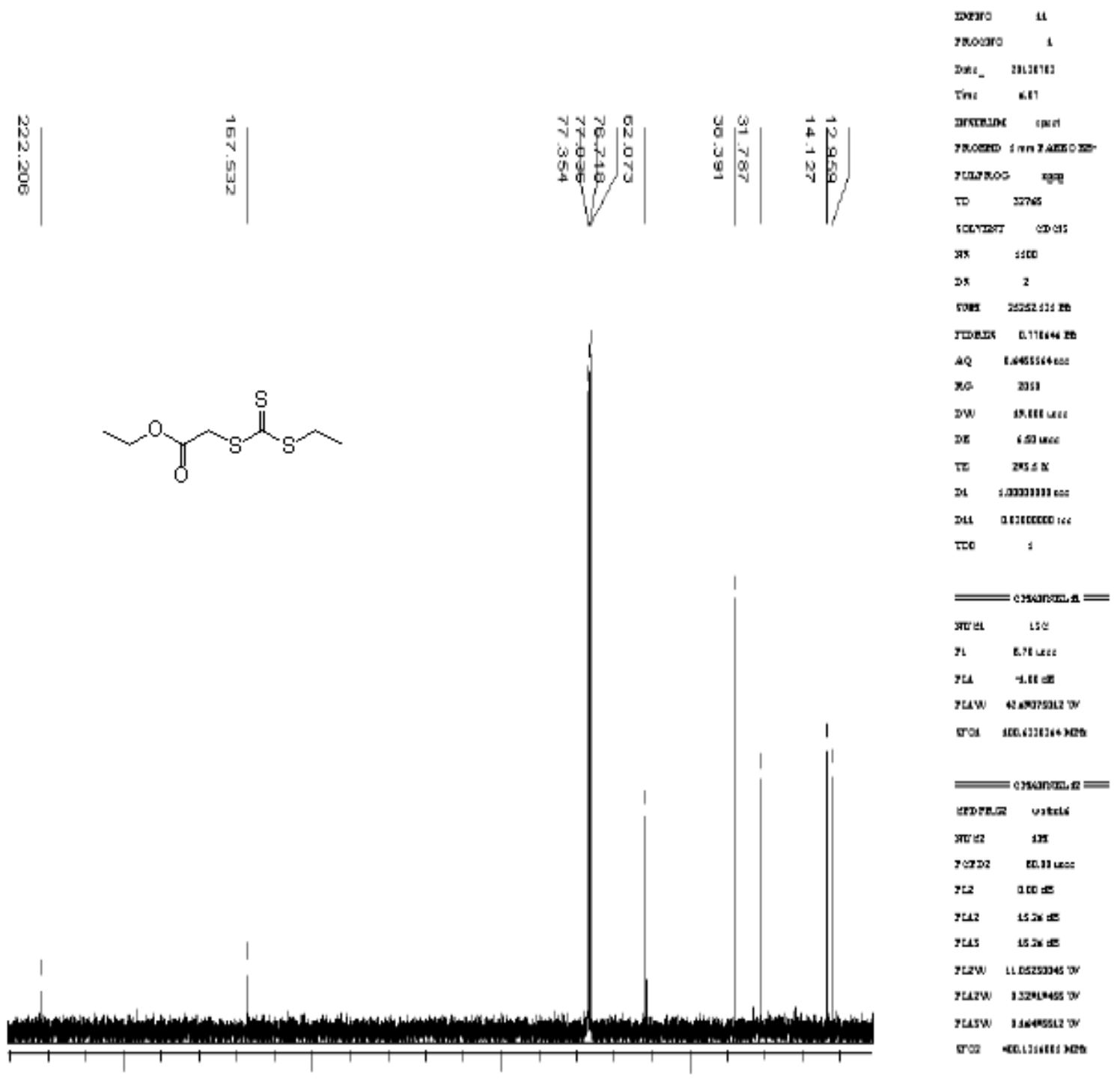




\section{Supporting Information}

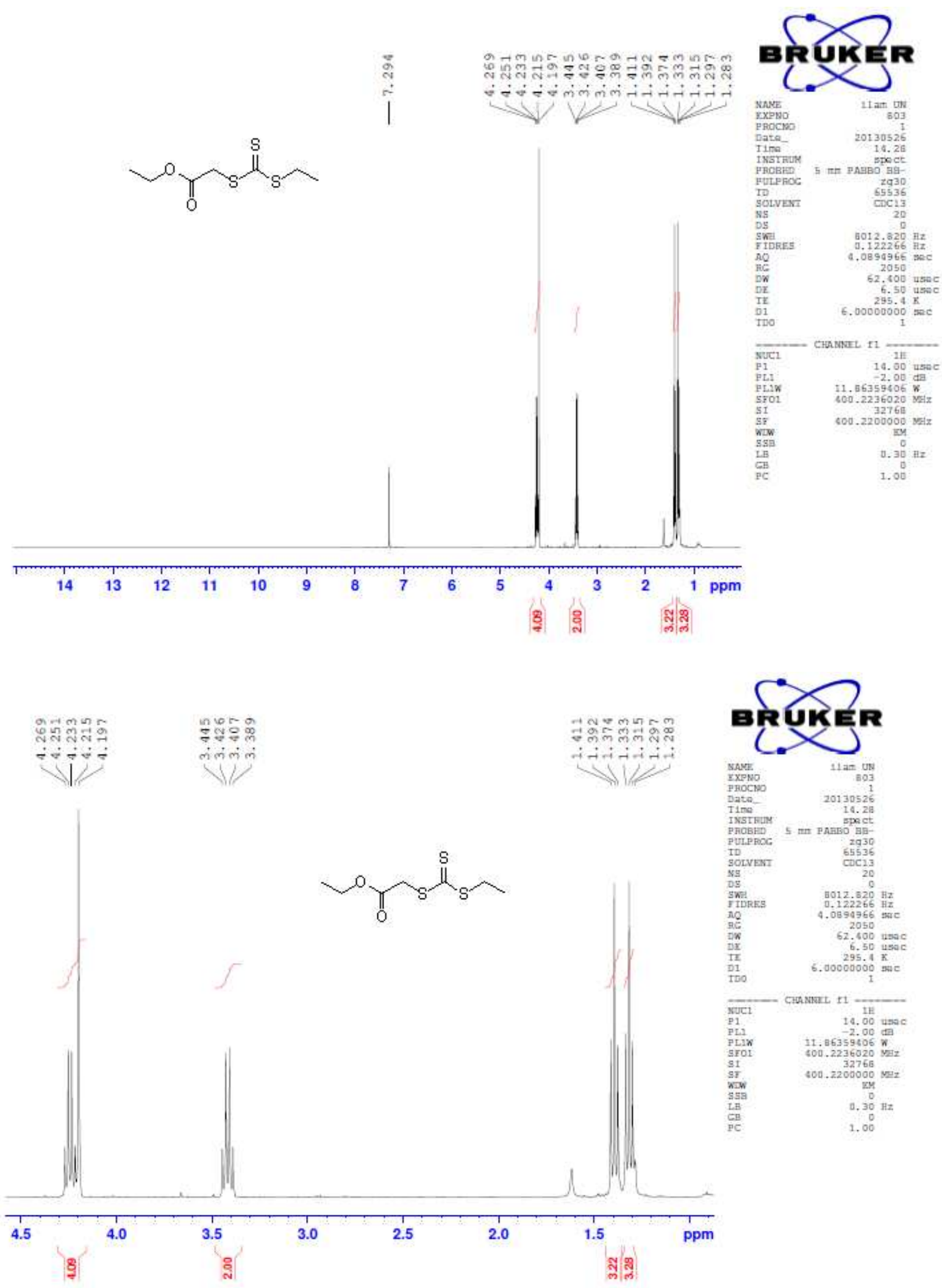


Supporting Information

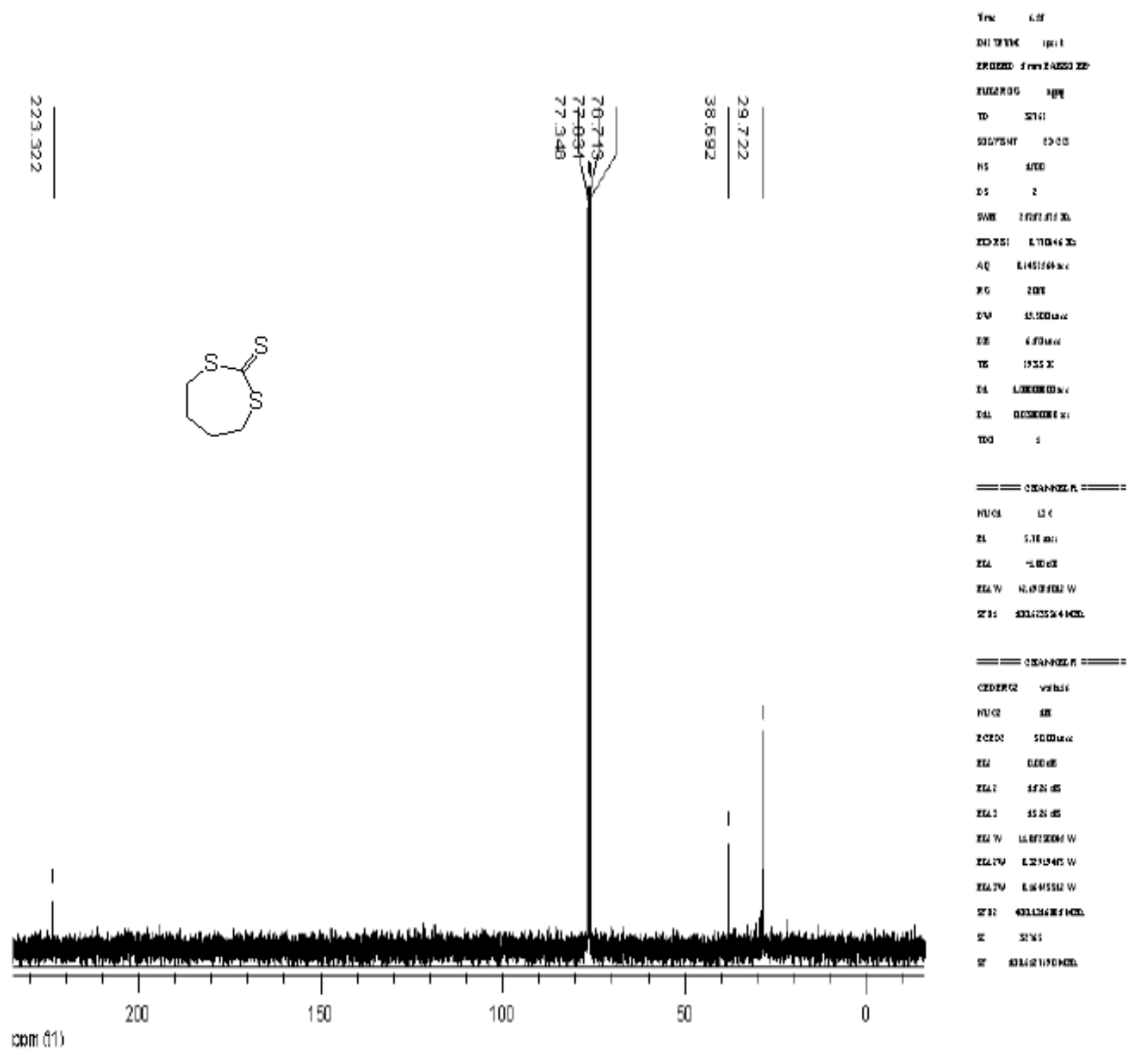




\section{Supporting Information}
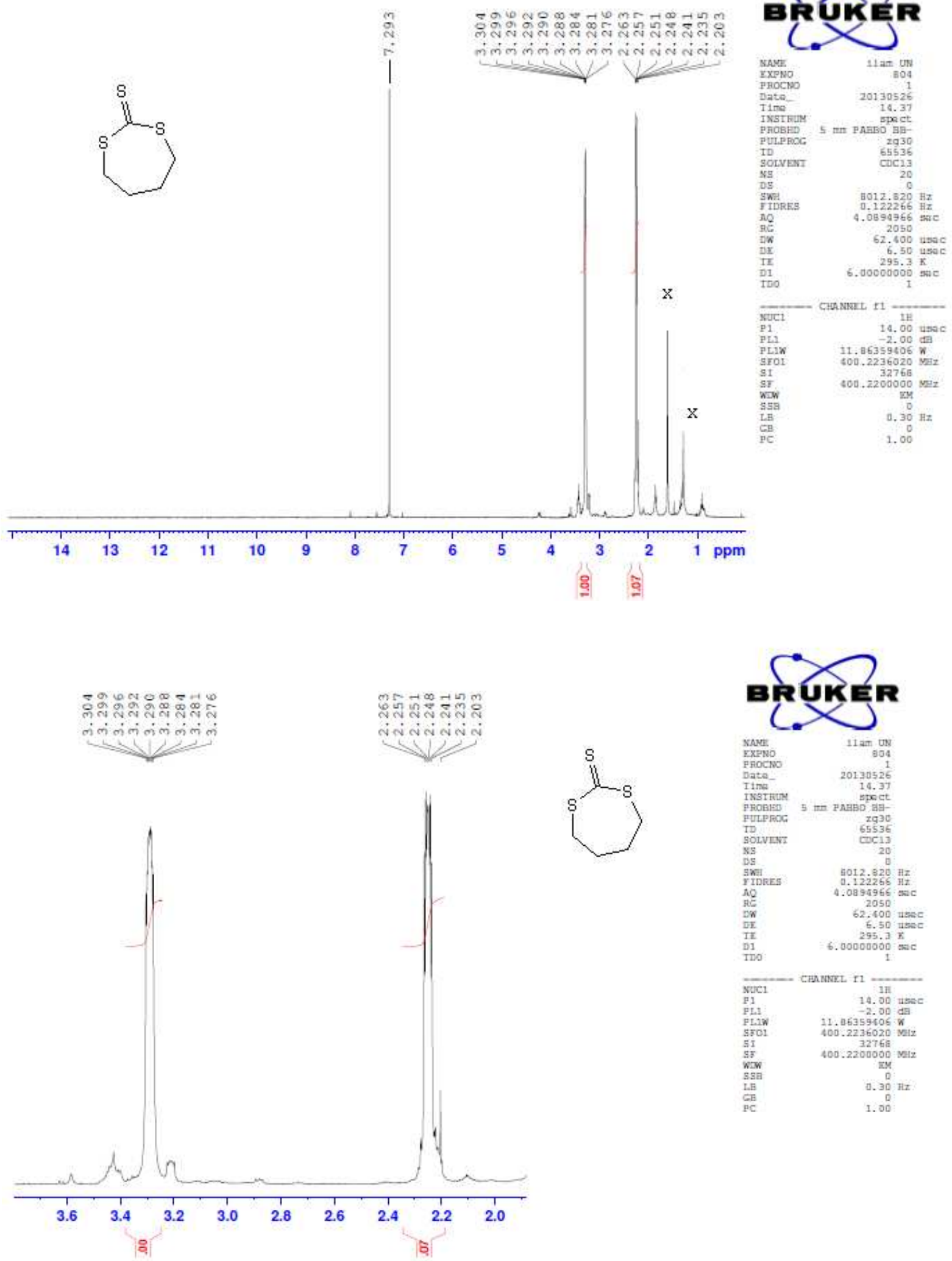
Supporting Information
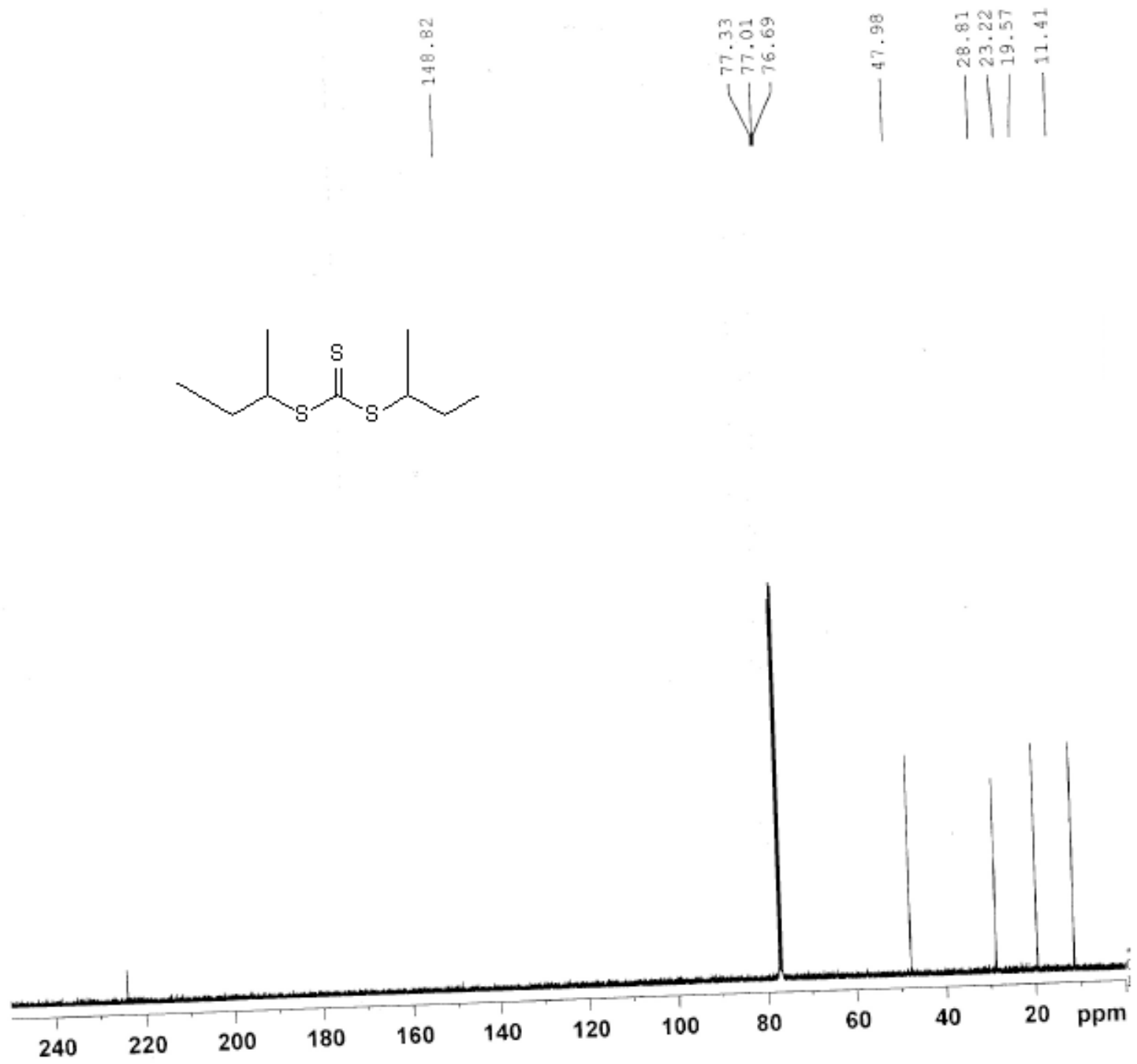
Supporting Information

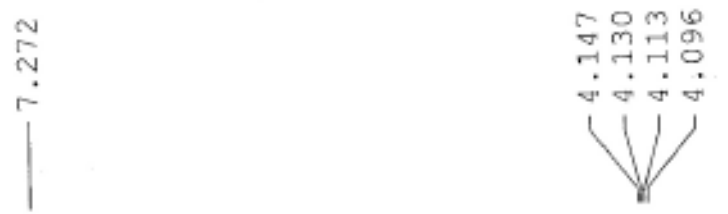

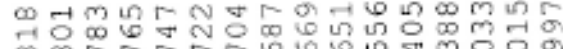

$\infty$ m r m r

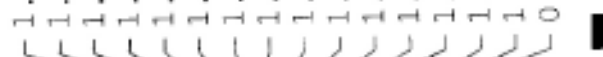

E
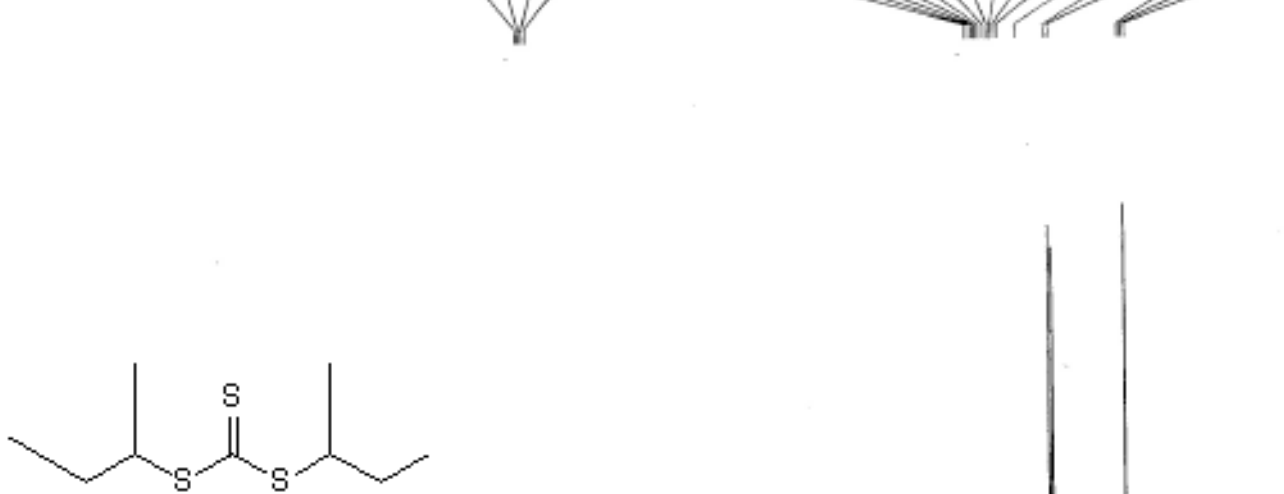

$\mathrm{Cu}$
NAI
EX
PR
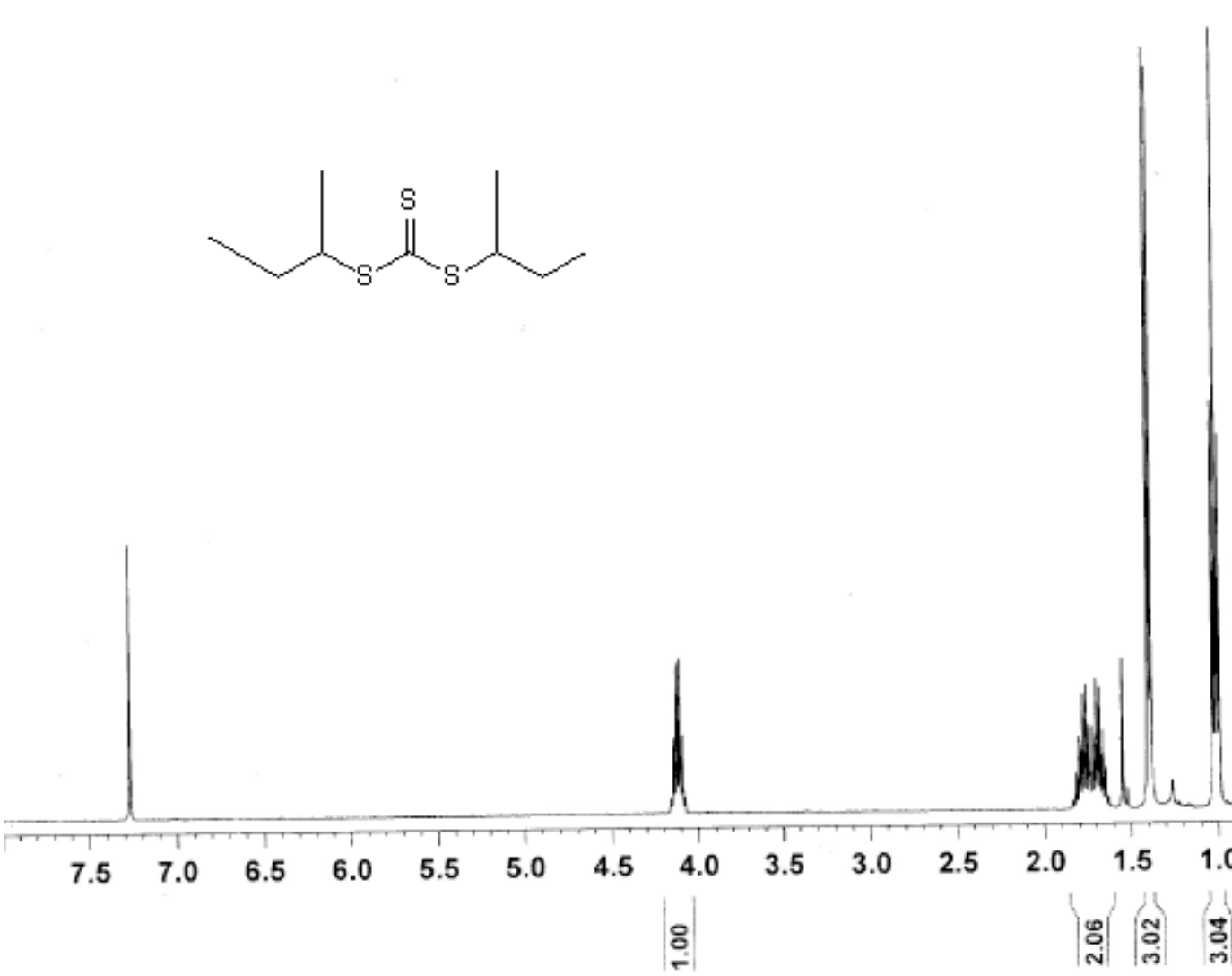

@

品 ( 
Supporting Information
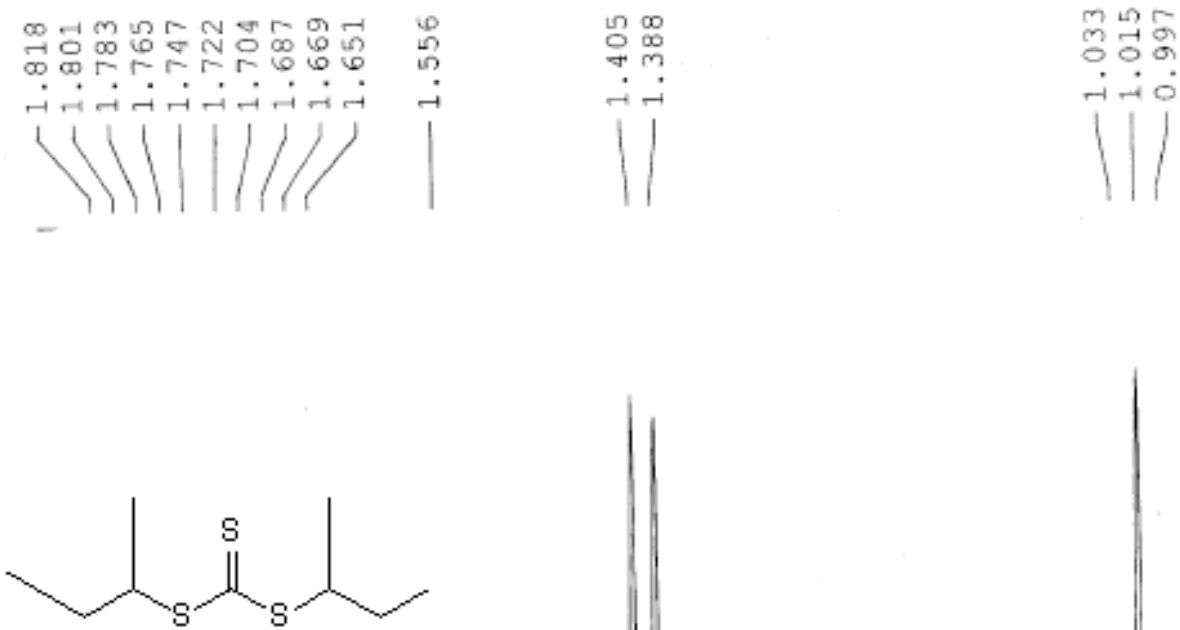

कㄷㅇㅇㅛ

NWVWV

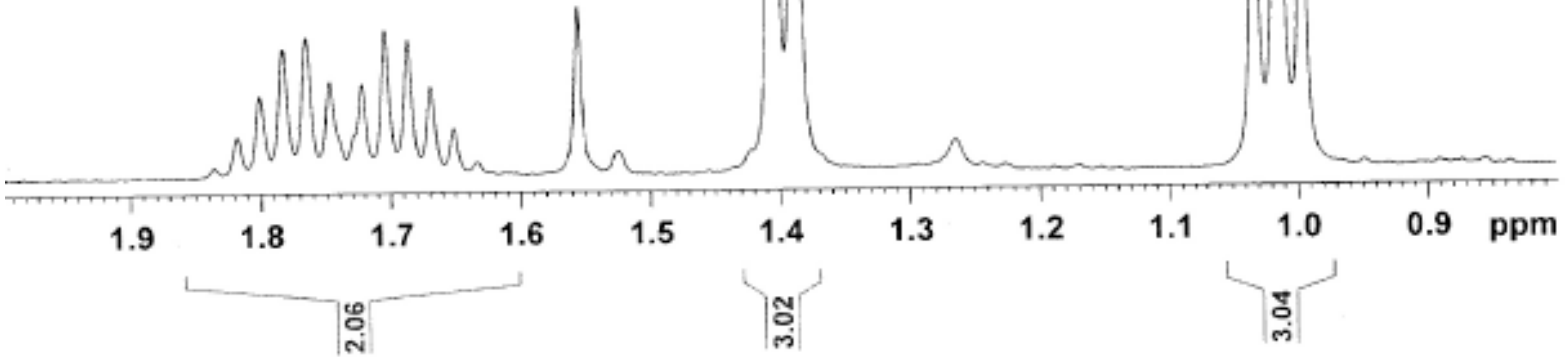


Supporting Information

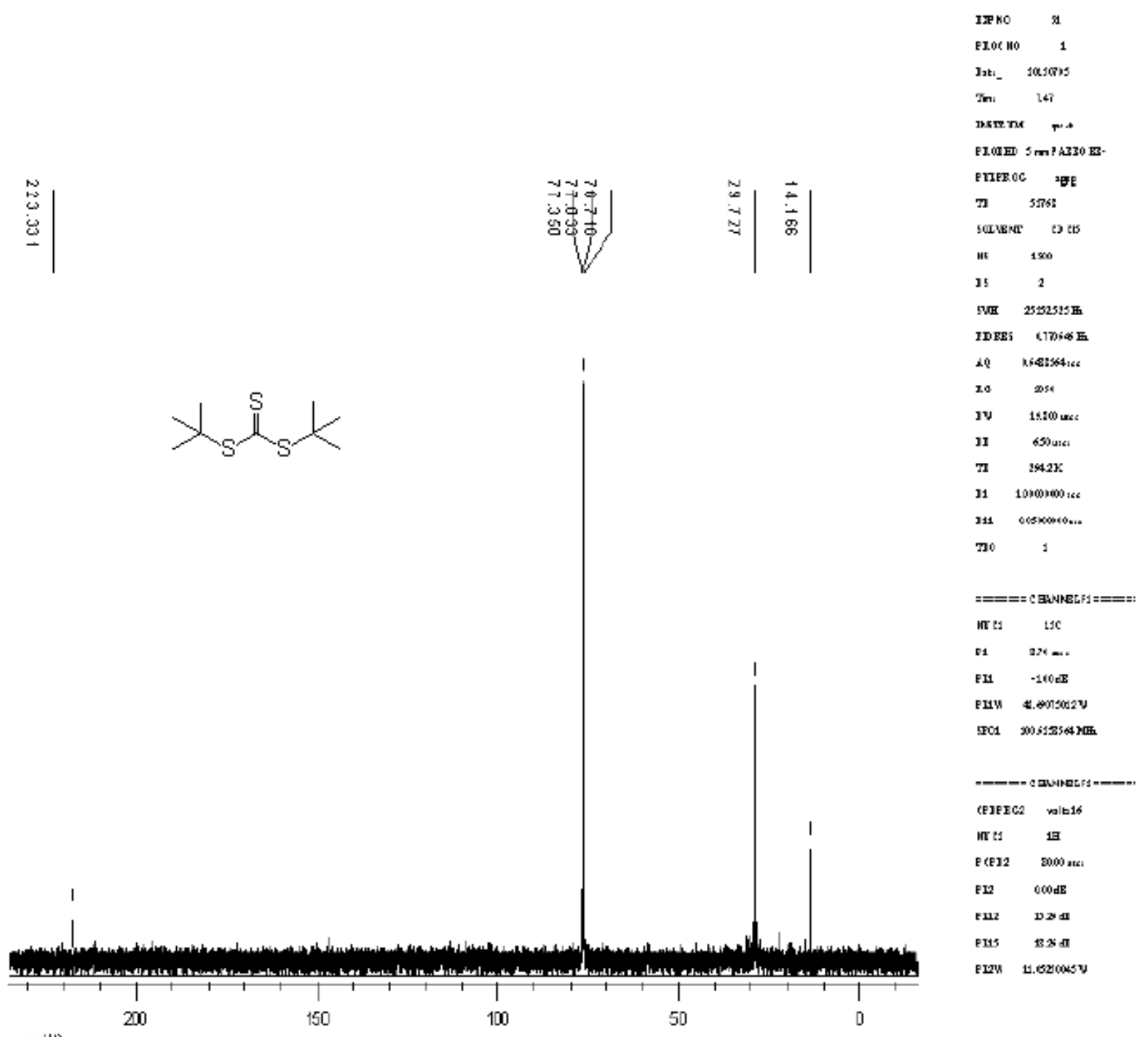


Supporting Information

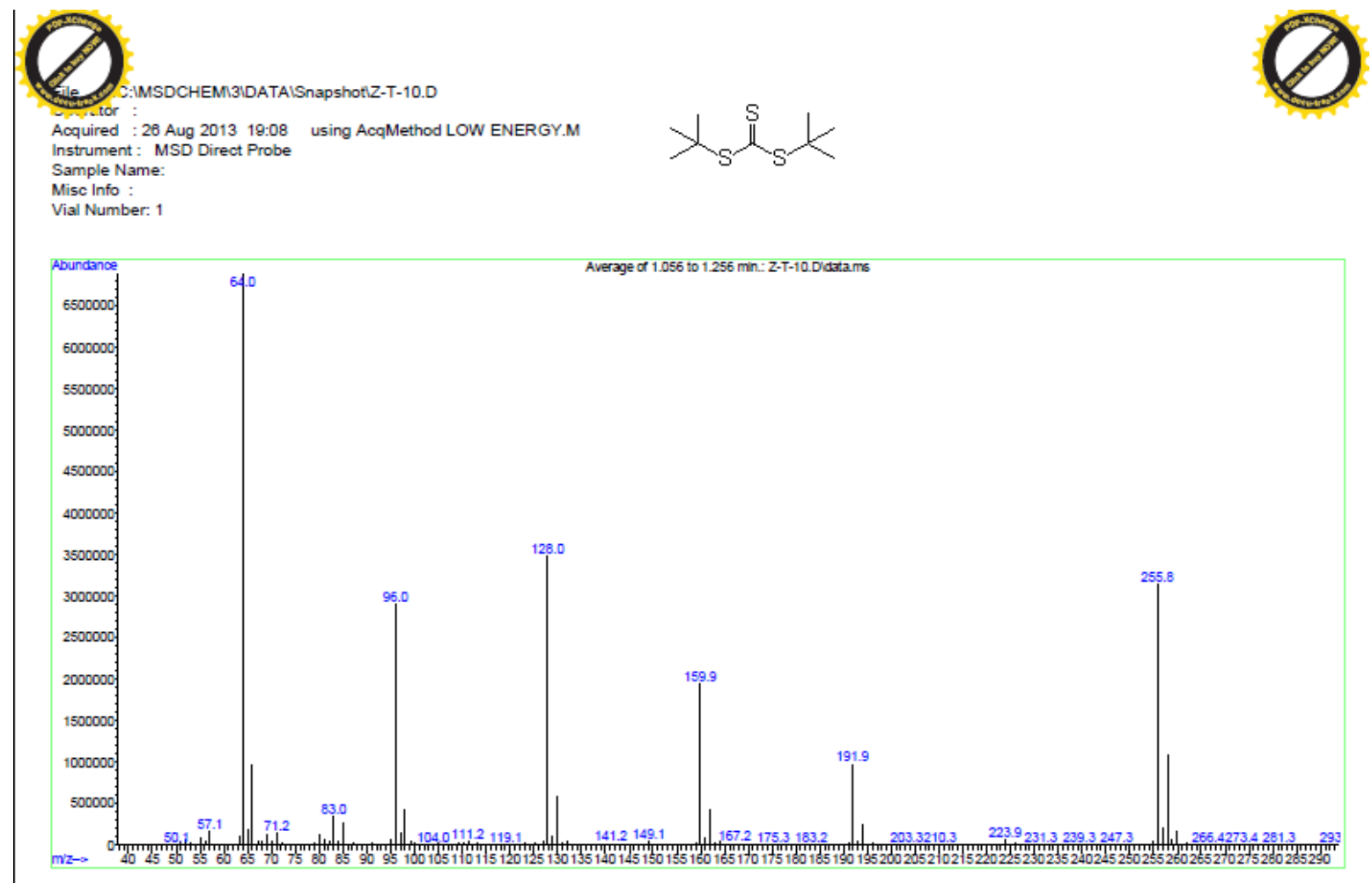


Supporting Information
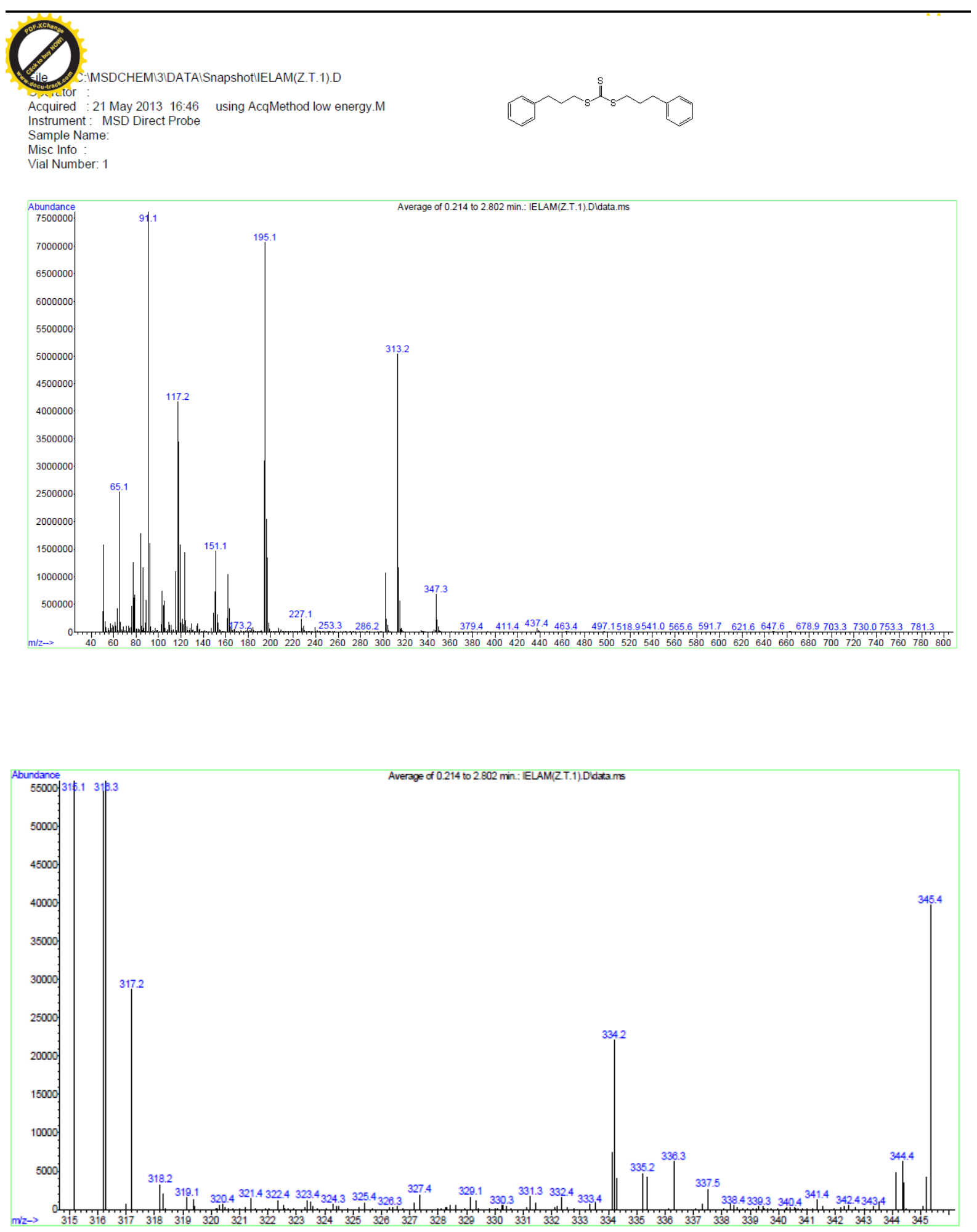


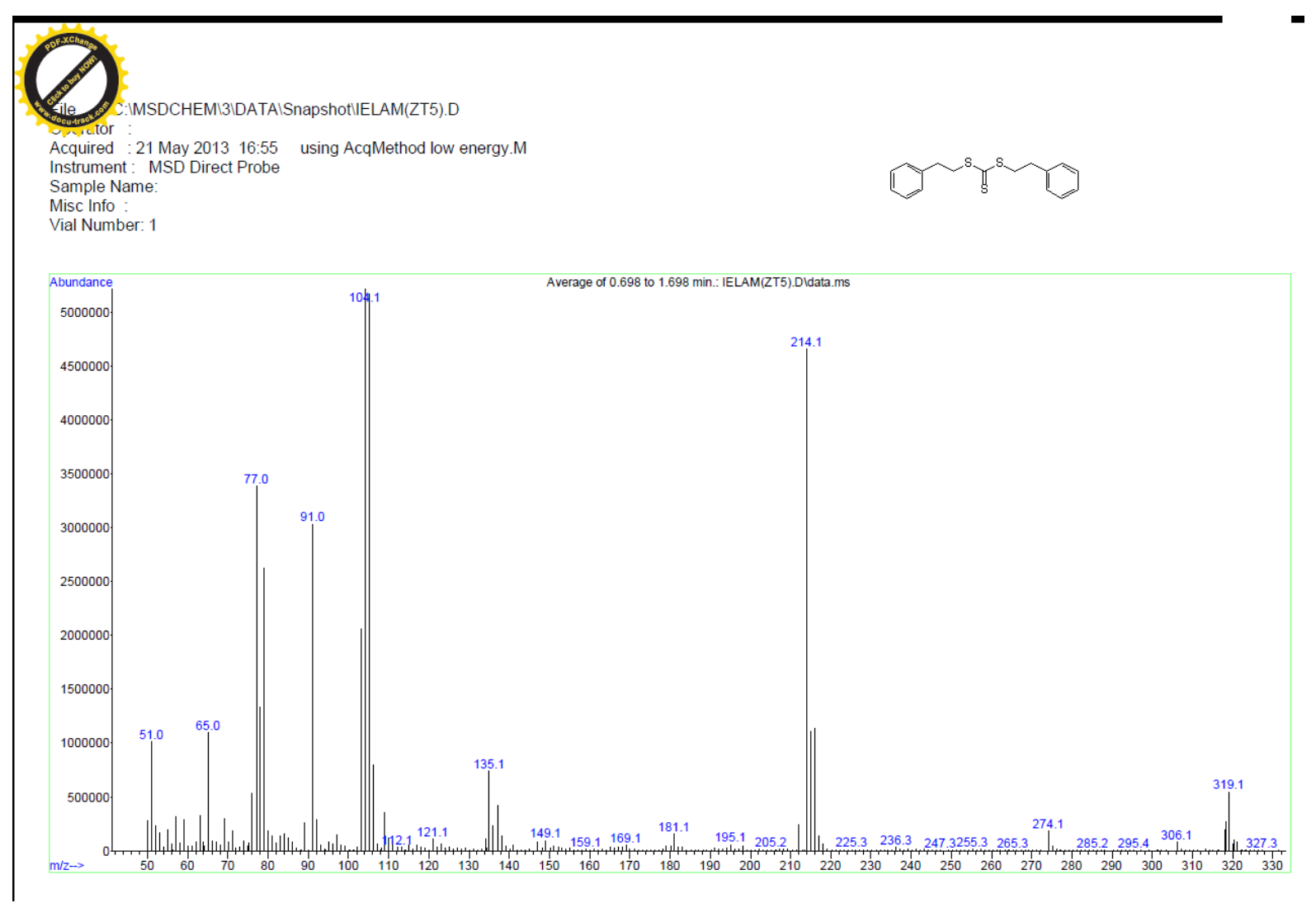

Vial Number: 1

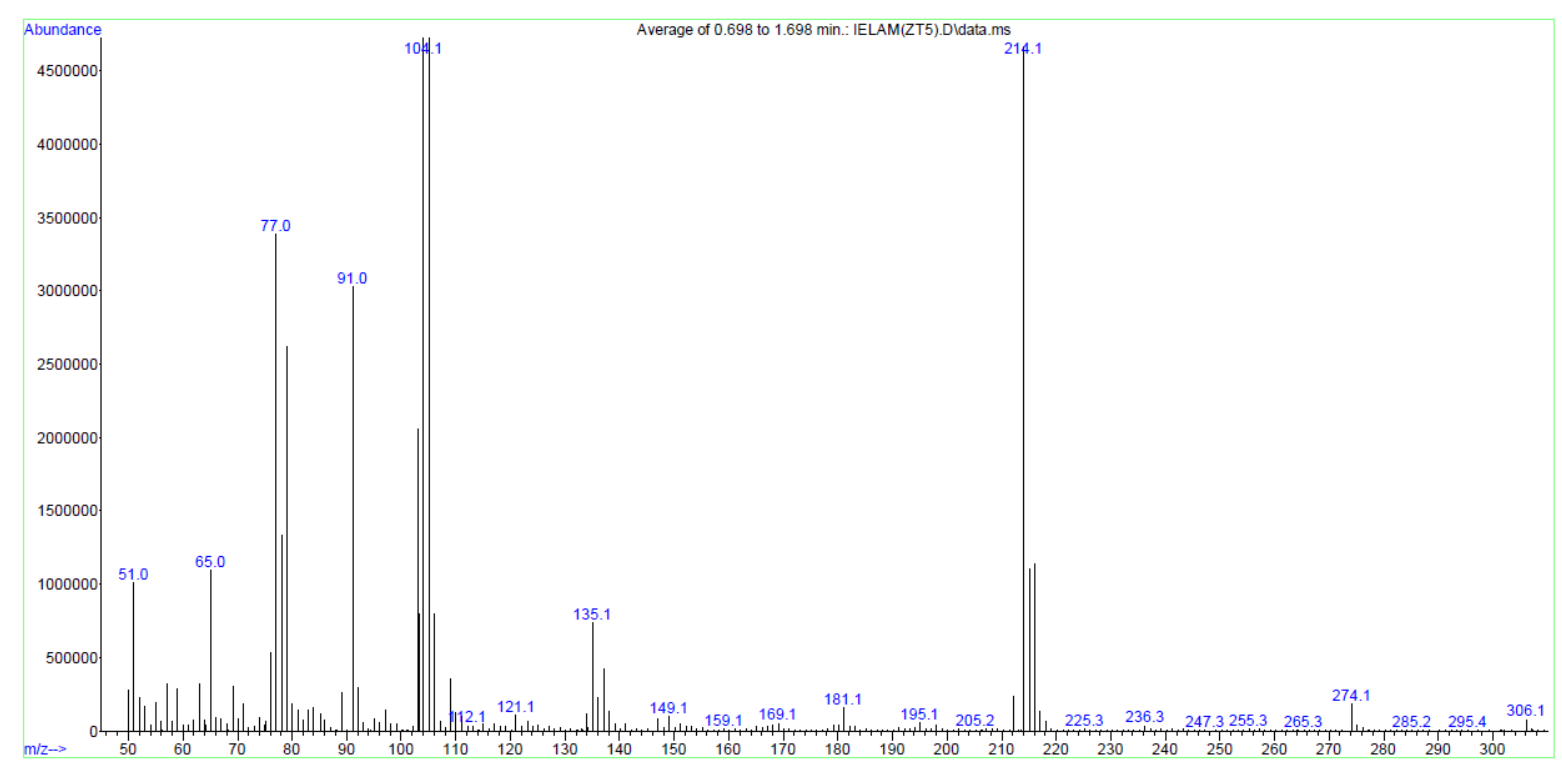




\section{Supporting Information}
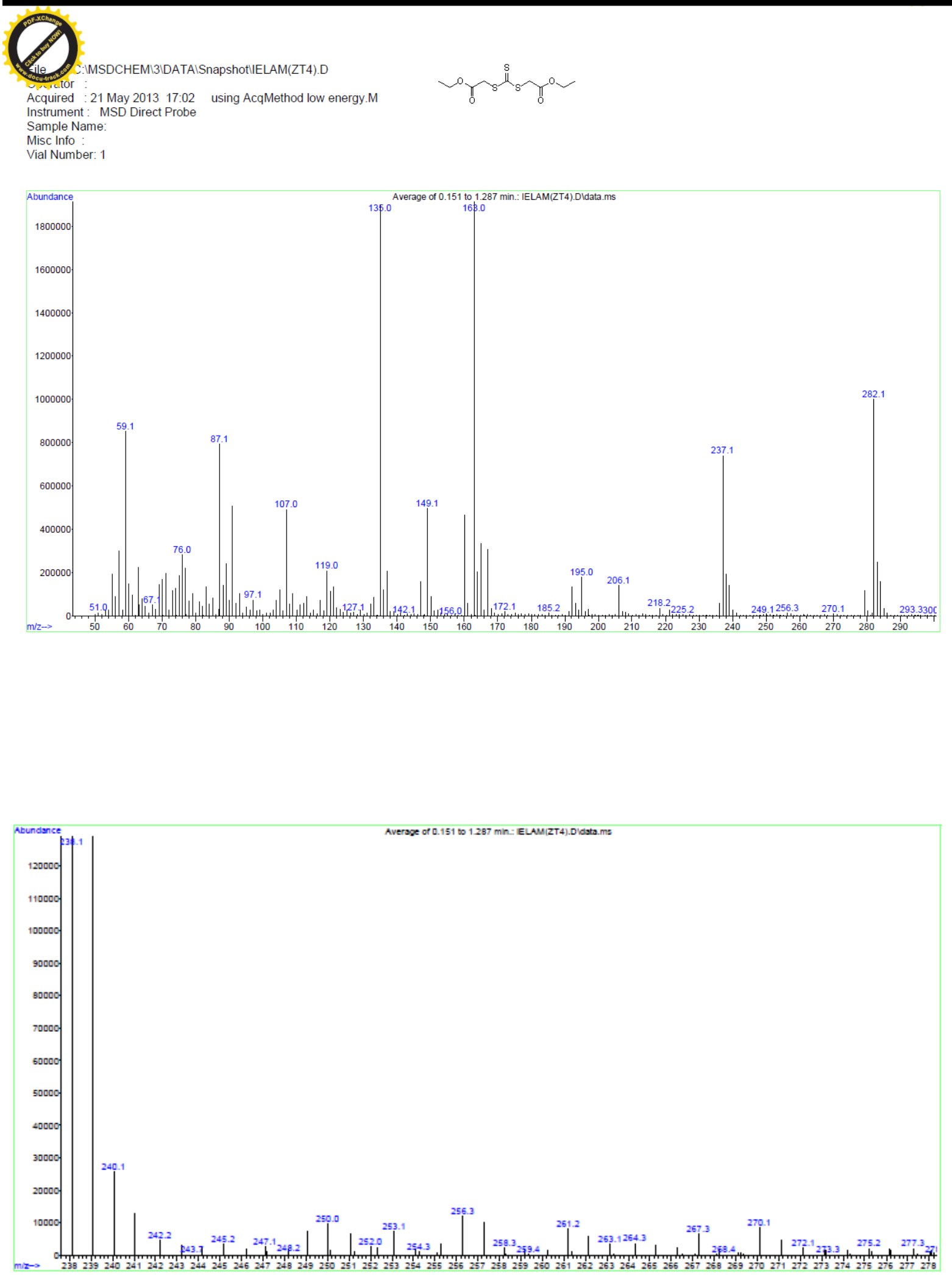


\section{Supporting Information}
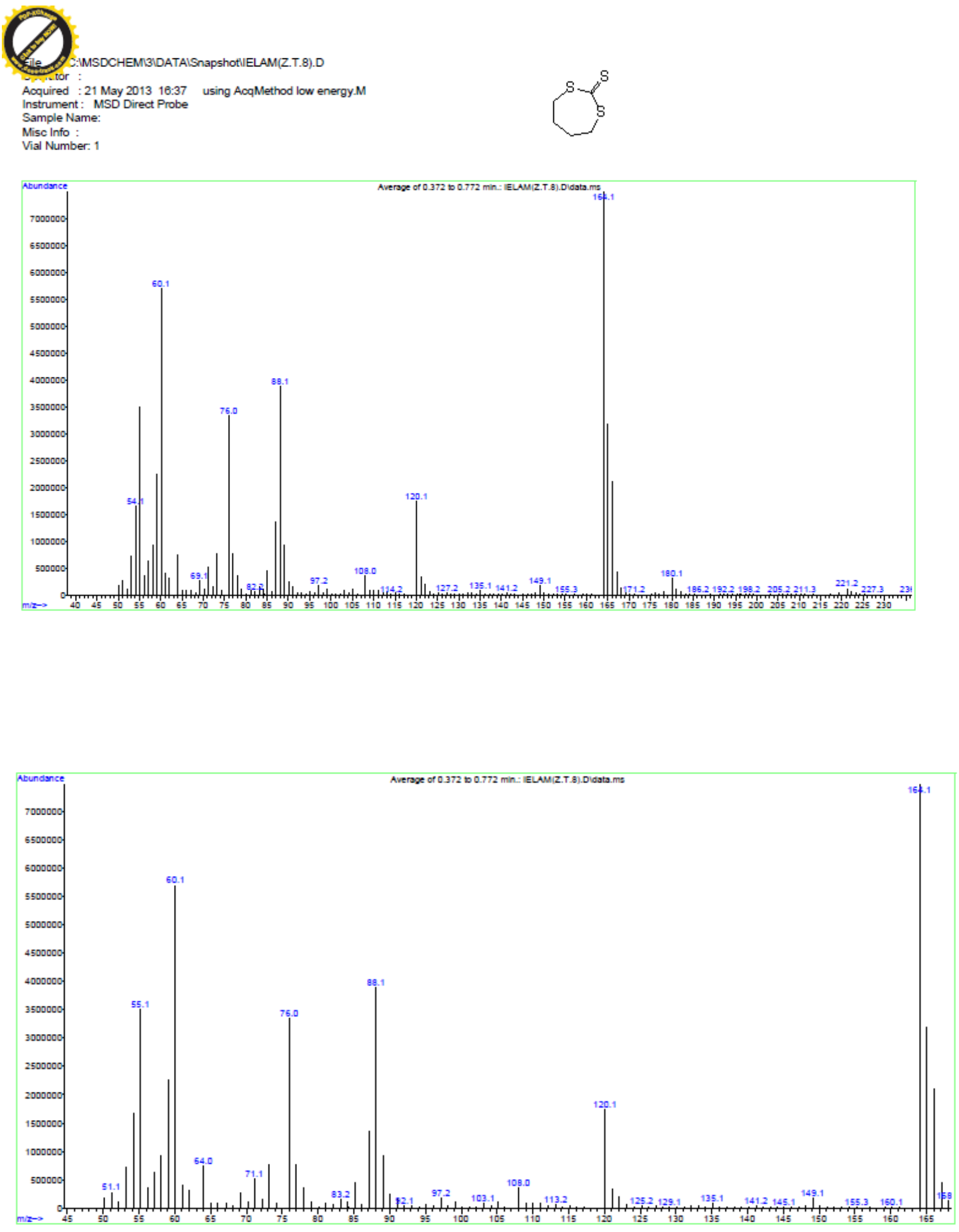

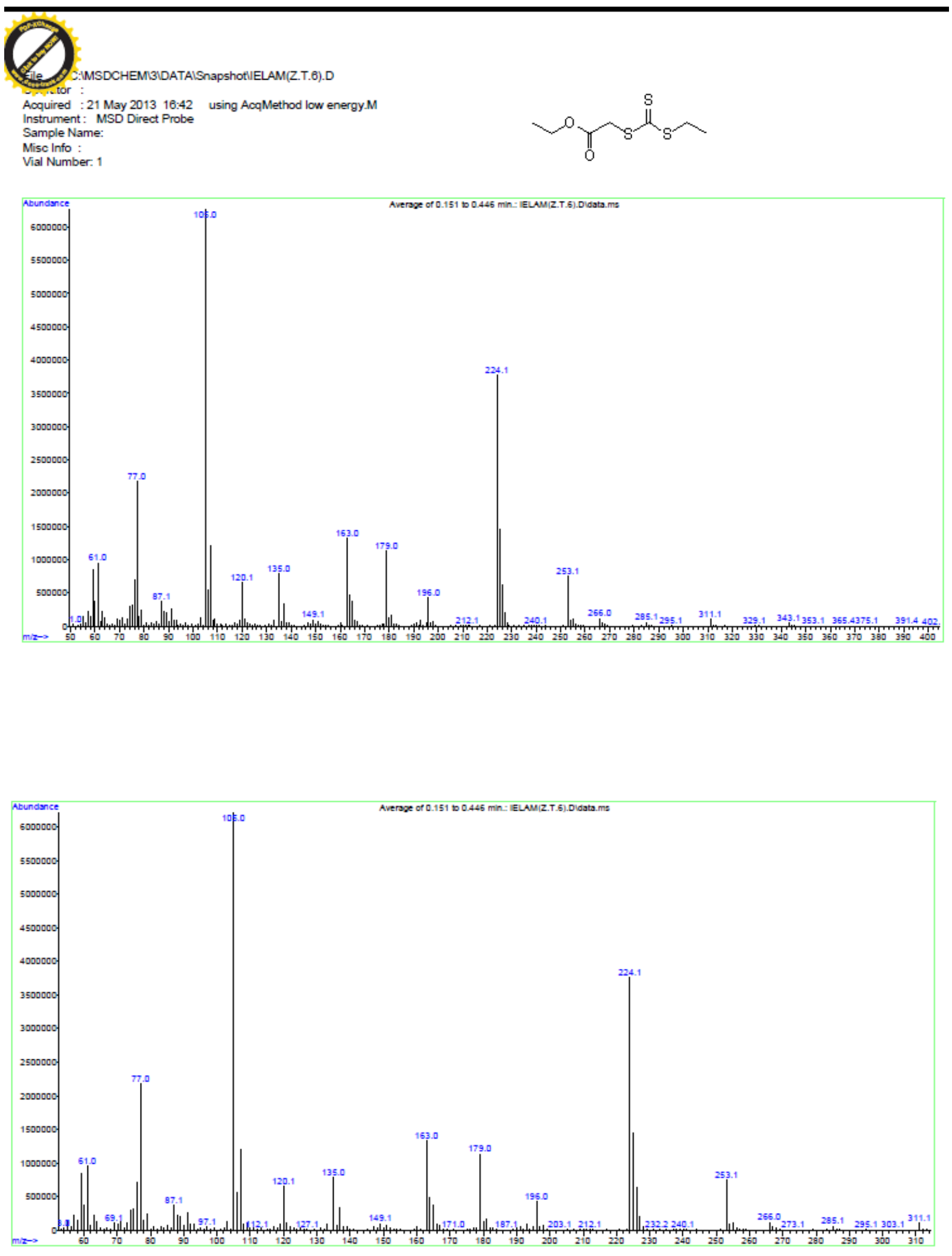